\title{
Optical Interferometry of Non-spherical Winds
}

\author{
Farrokh Vakili, Denis Mourard, Philippe Stee, and Daniel Bonneau \\ Observatoire de la Côte d'Azur, Fresnel-GI2T, F-06460 Caussols, France
}

\begin{abstract}
Optical Long Baseline Interferometry (OLBI) combined with spectral resolution is capable to sounding the stratification of luminous star winds. In this paper we review some few, yet unique results obtained on P Cyg and Be stars from OLBI observations. We also examine the prospects for studying hot stars winds offered by optical synthesis arrays presently under construction.
\end{abstract}

\section{Introduction}

High angular resolution has always opened up new vistas in astronomy and this is particularly true for studying the physics of hot luminous stars and their winds. While the HST has sharpened our view of the nebulosities around LBV's, WR and other exotic objects (see the superb results presented during this colloquium), zooming into the immediate surroundings of their central engines will need sub-mas spatial resolution only achievable by means of OLBI. Hopefully, the planned operation of more than 10 interferometers within 10 years, working at both the visible and IR wavelengths (for more details see SPIE proceedings 1998) will offer the tools to improving our understanding of the mechanisms producing massive stars photometric, spectrometric and polarimetric variability.

\section{Spectrally resolved interferometry: how it works?}

Michelson interferometers recombine the stellar light from widely separated telecopes in a central focal plane where the estimate of the so-called fringe visibility informs about the angular extent of the source in the direction of the interferometric baseline. The fringes are classically analysed through a chromatic filter which has the property to average the angular extent of the source over the filter bandpass. In this process and in the case of a 2 telescope interferometer any information about the position and asymmetry of the celestial source is lost unless one can record simultaneously the fringe signal from a pointlike reference source within the interferometric field of view. It was recognized in early days of modern OLBI (Labeyrie 1980) that measuring the fringes of an emission line star through a spectrograph is similar to having such a reference provided the star is unresolved at the continuum wavelengths. In this way it becomes possible to derive both the size 
and position of the emitting regions around a star as a function of Dopplershift accross its spectral lines. We call this technique Interferometric Doppler Imaging (IDI) by analogy to classical spectroscopic Doppler-imaging where the temporal changes of line profiles are replaced by relative spatial positions obtained from the interferometer. For technical aspects of IDI we invite the reader to see Vakili et al. 1997 and recall that IDI can achieve sub-mas superresolution on the kinematics of hot star winds with optical interferometers using decametric baselines.

\section{P Cyg august 4th 1994}

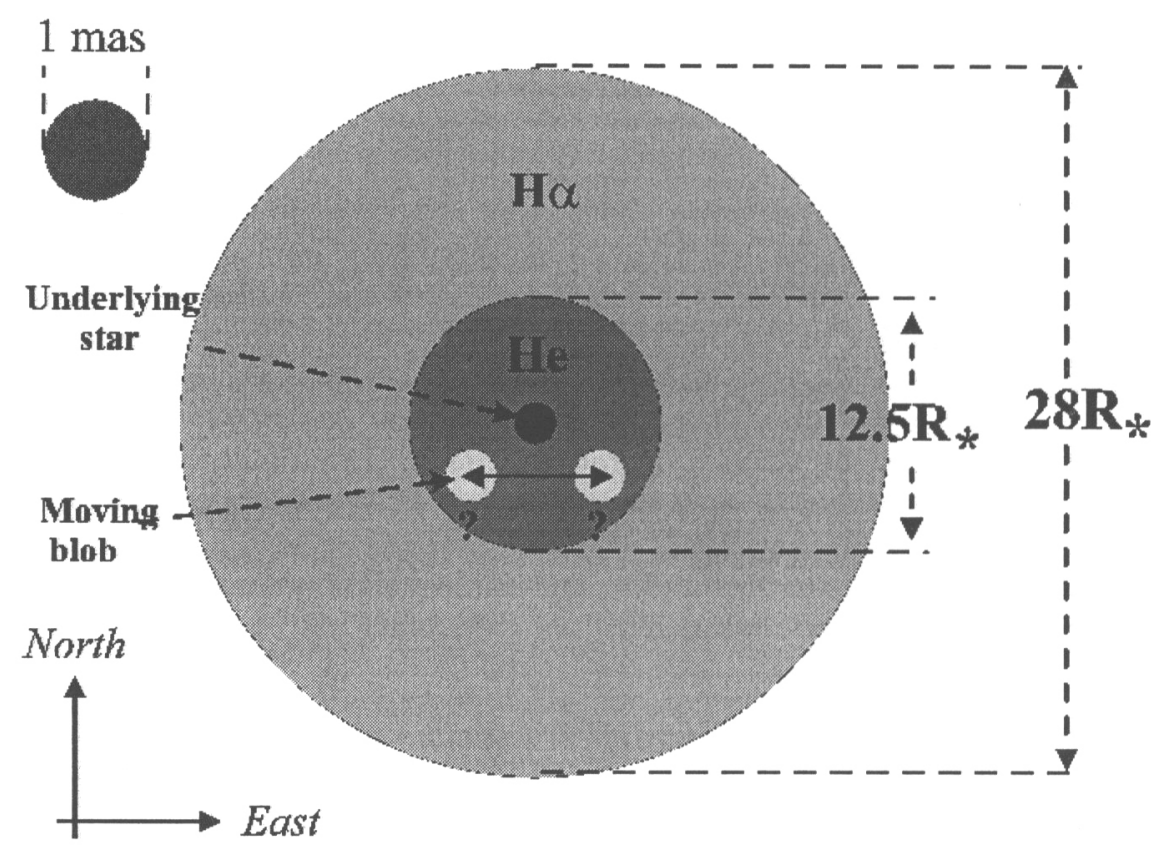

Fig. 1. Schematic morphology of P Cyg according to GI2T spectrally resolved interferometry in '93. The $\mathrm{H} \alpha$ and HeI6678 emitting envelopes given in units of stellar radius $\left(76 \pm 15 R_{\odot}\right.$ corresponding to 0.4 mas for a distance of $1.8 \mathrm{kpc}$ ). The projected position of the $\mathrm{H} \alpha$ emitting blob detected by the GI2T is at 4 stellar radii to the south of the central star BUT its E.-W. position (noted by ?) is unknown. 


\section{Asymmetry in the wind of P Cyg}

The prototype LBV star P Cyg constitutes a favorite target for spectrally resolved OLBI. Following earlier attempts, the GI2T-team was able to successfully observe P Cyg in August,1994 in both $\mathrm{H} \alpha$ and HeI6678 lines with an effective spectral resolution of $1.7 \mathrm{~A}$. Based upon robust calibrations on a number of reference stars, Vakili et al. 1997, estimated the equivalent uniform disk diameter of $\mathrm{P}$ Cyg in $\mathrm{H} \alpha$ as $5.52 \pm 0.47$ mas corresponding to 28 stellar radii at a distance of $1.8 \mathrm{kpc}$. By sampling the interferometric signal across $\mathrm{H} \alpha$ using spectral windows of $3.4 \mathrm{~A}$ wide, starting from the blue and finishing in the red wings of the line, an asymetrical structure was discovered at $\mathrm{RV}=-208 \mathrm{~km} \mathrm{~s}^{-1}$. The north-south projected position of this structure, presumably a bright blob in P Cyg's wind, was found at 4 stellar radii to the south of the central star (Fig. 1). Vakili et al. gave also a rough estimate of the HeI6678 emitting envelope as 12.5 stellar radii on the order of the distance of the detected blob and argued about possible relation of asymetries at the basis of $\mathrm{P}$ Cyg wind and the fine structures at larger distance from the central star detected by HST coronography (Nota et al. 1995).

\section{Interferometry of Be star disks}

OLBI observations of Be star disks have been made so far by 3 different interferometers (see Vakili et al. 1994 and references therein). Following earlier observations from the I2T and GI2T interferometers in the southern France (Thom et al. 1986, Mourard et al. 1989) Quirrenbach and colleagues carried a systematic survey of the brightest northern hemisphere Be stars up to the 4th visual magnitude (see Stefl's review in these proceedings). The survey was made using earth-rotation synthesis possibilities offered by the Mark III interferometer at Mount Wilson (Quirrenbach et al. 1997). These remarkable observations proved that the extent of Be star envelopes exceed tens of stellar radii and are indeed flattened disks with an excellent correlation of their elongation positionnal angle and spectropolarimetric measurements.

The Mark III group gave the extent of Be star disks through a $1 \mathrm{~nm}$ filter which was narrower than their $\mathrm{H} \alpha$ emission for a number of them, thus masking their envelope highest RV regions. Also no information was given on the relative position and extent of emitting iso-radial velocity regions inside the envelope. Thus, despite its excellent sensitivity, Mark III could not determine any departure from azimuthal symetry of Be star equatorial disks. This shortcoming can be particularly misguiding in the case of binary Be stars ( $\phi$ Per) or those objects which exhibit indirect evidence for azimuthal asymetry ( $\zeta$ Tau, see below) or co-rotating structures inferred from X-ray and UV spectroscopy (Smith et al. 1998). Despite this, Mark III observations provide a solid ground upon which future interferometric work aiming to map Be star disks by means of aperture synthesis imaging can be built. 
Based on a radiative transfer model, more recent studies of $\gamma$ Cas in $\mathrm{H} \alpha$ (Stee et al. 1995), $\mathrm{H} \beta$ and HeI6678 (Stee et al. '98) give the most complete picture of $\gamma$ Cas envelope constrained from GI2T spectrally resolved observations (Stee et al. these proceedings).
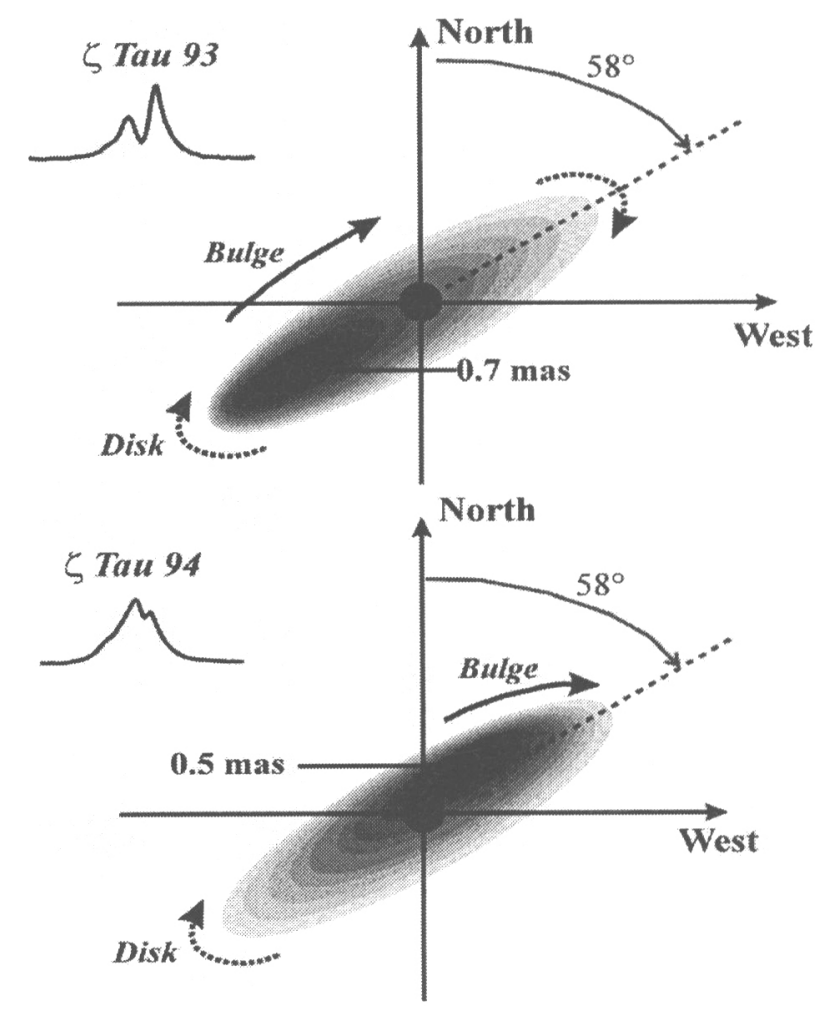

Fig. 2. $\zeta$ Tau's long term variability representation according to GI2T. In November '93 $\mathrm{H} \alpha$ has a $\mathrm{VR}=.57$ profile and the extra $\mathrm{R}$ emission in the disk originates in a region whose N-S projected position is at 0.7 mas South of the central star. In October ' $94 \mathrm{VR}=1.26$ and this region has a $\mathrm{N}$ - projected position at 0.5 mas North of the central star. The elongation angle of the envelope projected on the sky is at $58^{\circ}$ West from the North. The curved arrows marked Disk and Bulge depict the absolute direction of rotation of $\zeta$ Tau's disk and the density wave inside this disk (prograde motion).

\subsection{Polarimetry AND interferometry of $\gamma$ Cas}

In principle there is no obstacle to equip the focal instrument of an interferometer by polarimetric optics. Added to spectral resolution, a polarimeter 
enables OLBI to study the extent and position of emitting regions of the wind as a function of wavelength AND of polarization, either linear or circular. Pilote experiments of this kind on the GI2T (Rousselet-Perraut et al. '97) have been carried on $\gamma$ Cas in '94 using quite simple polarizing filters. Although these observations failed to detect any noticeable effect between linear polarizations of this star, they determined its angular size in natural light at $\lambda=660 \mathrm{~nm}$ and $\Delta \lambda=3.4 \mathrm{~nm}$ ) as $2.9 \pm 0.5$ mas. This extent is somehow larger than the value usually admitted in the litterature (Stee et al. 1995). If confirmed by future interferometric observations they it would mean that the continuum emission of the envelope (free-free, free-bound, scattering) would be larger than classically thought (Waters et al. '92).

The difficulty of spectro-polarimetric interferometry is due to their optical complexity which demand numerous and stringent calibrations of internal effects in order to attain the desired and unbiased accuracies to determine any polarization effect from stellar origines. This difficulty is somehow counterbalanced by the interferometric angular resolution of local polarized structures of the star's wind otherwise averaged over its extent when using classical spectropolarimetry at low spatial resolution. To better understand this property, one can think of the special case of a dipolar magnetic star having identical rotation and magnetic axes perpendicular to observer's line of sight. No Zeeman signature could be observed by spectropolarimetry whilst an interferometric signal could in principle be detected from the different spatial locations of $\sigma_{+}$and $\sigma_{-}$components of a the star across its spectral lines.

\subsection{The case of the Be eclipsing binary $\beta$ Lyr}

The Be star $\beta$ Lyr, also known for its strong radio emission, is a puzzling eclipsing binary $(\mathrm{B} 0 \mathrm{~V}+\mathrm{B} 6 \mathrm{II} \mathrm{p})$ whose resolution constitutes a challinging problem to optical interferometers. In a coordinated multi-site/technique campaign organized on '94, the GI2T-team (Harmanec et al. 1996) could find the existence of jet-like structure perpendicular to the accretion disk around the BOV component. This conclusion came from the fact that, although the $\beta$ Lyr complex remained unresolved as a binary system by the GI2T, its $\mathrm{H} \alpha$ flow was partially resolved in the north-south direction without any net dependence on the orbital phase.

In a recent paper the University of Winconsin team (Hoffman et al. 1998) reported the analysis of UV and visual spectropolarimetric data on $\beta$ Lyr between '92 and ' 94 . They found the continuum polarization to be at $90^{\circ}$ of the Balmer lines polarization which remained constant whatever the orbital phase thus confirming the bipolar nature of the $\mathrm{H} \alpha$ emission discovered by the GI2T-team. The complex morphological picture of $\beta$ Lyr derived from these observations predicts significantly different spatial locations of the emitting regions in the continuum and spectral lines AND as a function of polarization and orbital phase. Indeed these locations could be determined from IDI 
observations on the GI2T which has a Wollaston-based polarimeter coupled to its spectrograph (Mourard et al. 1998).
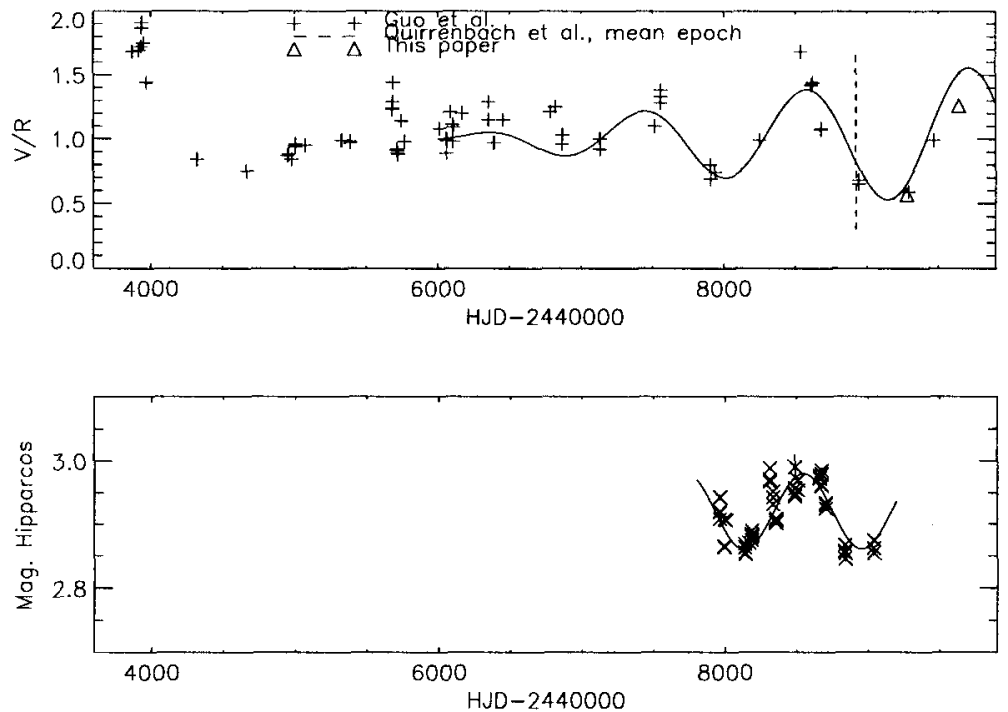

Fig. 3. Top: long term $\mathrm{H} \alpha \mathrm{V} / \mathrm{R}$ variability of $\zeta$ Tau. The continuous line corresponds to the fit of an amplifying sine wave after 1988. Bottom: photometric data from the Hipparcos mission. Note the relatively good correlation between the $V / R$ and photometric cyclicities.

\subsection{Interferometry and long term Be variability}

One of the long standing problems of Be stars (Telting et al. 1994) has been their observed cyclic V/R variability (V/R: Violet to Red intensity ratio of spectral emission profile wings). The $V / R$ cycles are on the order of a few years, rarely exceed ten years and can be followed/preceeded by active periods where the Be star presents LBV-like eruptions (Marlborough 1997). Plausible scenarios accounting for $\mathrm{V} / \mathrm{R}$ cyclicity have only recently emerged from theoretical studies of global oscillations of Be star equatorial disks (Papaloizou 1992, Okazaki 1997). Following our earlier suggestion (Vakili et al. 1994) to check such theories against OLBI observations and using GI2T interferometry of $\zeta$ Tau on November '93 and October '94, we determined the apparent motion of a prograde density wave in the equatorial disk of this $\mathrm{Be}$ shell star (Fig. 2). For our epochs of observation $\zeta$ Tau presented $\mathrm{H} \alpha \mathrm{V} / \mathrm{R}$ values of 0.57 and 1.26 (derived from the GI2T's spectrograph itself) which 
nicely agreed with those of Guo and co-workers' spectroscopy until '93 (Guo et al. 1995). Using these data, we derived a 3.1 years cycle for $\zeta$ Tau's V/R variability in the range of the predicted values by the one-armed oscillation theory. We used the $58^{\circ}$ elongation angle of $\zeta$ Tau given by the Mark III interferometer (Quirrenbach et al. 1997) and phasing our interferometric data of '93 and ' 94 with the V/R cyclicity, determined the absolute positions of the enhanced density region across the equatorial disk of this Be shell star. The scaled geometry of $\zeta$ Tau projected on the sky plane is depicted in Fig. 2. Our GI2T findings agree surprisingly well with Hipparcos photometric data obtained during '92 and '93. Indeed, the partial eclipse expected to occur when the high density equatorial region passed behind the central star of $\zeta$ Tau, thus increasing its apparent magnitude (Mennickent et al. 1997) is visible on Hipparcos wide band photometry (Fig. 3).

This isolated result clearly shows that patrol OLBI observations must be applied to other objects, specially to pole-on Be stars like $\eta$ Tau, for which V/R variability cannot, or very hardly, be detected by spectroscopy. However, in order to interpret OLBI observations in terms of physical parameters of these stars, adequate models including hydrodynamics and radiative transfer coupling should produce interferometric observables from monochromatic maps across emission lines (Stee et al. 1995).

\section{Discussion}

The few results outlined above demonstrate that a number of important discoveries can be foreseen from the operation of optical synthesis arrays like the VLTI, CHARA and the Hawaii Keck interferometer in the coming decade. Meanwhile, the use of a full-fledged GI2T and its spectro-polarimetric focal instrument should bring new insights to the understanding of hot luminous stars and the physics of their winds. Hereafter is a non exhaustive list of problems that OLBI could adress:

a)Determination of the size of Be underlying (flattened?) stars: this is possible by spectrally resolved interferometry of photospheric absorption lines using earth-rotation synthesis (a 3 telescope interferometer is more efficient). b)Detailed imaging of Be star equatorial disks and their kinematics: this is better done using the dignosis value of optically thin FeII emission lines (Hanuschick '96) and also carrying simultaneous visible and infrared OLBI. c) The role played by NRP and magnetic activity in the variability of massive star winds: this is possible with mas resolution to sound the inner regions of the wind in Balmer series and spectral lines of $\mathrm{He}, \mathrm{Si}, \mathrm{O}, \mathrm{C}$, etc..

d) Multiplicity of massive stars: interferometry can detect unseen companions and "weight" the individual masses. For close binaries the detailed geometry of the system can be mapped as a function of orbital phase to better understand mass exchange mechanisms for instance. 
e) Direct detection of inhomogenities at the basis of massive star winds (like for P Cyg): this probably needs OLBI observations to be alerted by spectro/photometry to follow the spatial change and location of the inhomogeneity until its effect, if any, becomes observable at lower spatial resolutions achievable by HST or adaptive optics on $4-8 \mathrm{~m}$ class ground telescopes.

f)Last but not least, for a number of luminous hot stars OLBI can directly determine parameters like $R$ and $L$ of the Wind-Momentum Relation $\dot{M} v_{\infty} R^{0.5} \propto L^{x}$ as defined by $\mathrm{R}$. Kudritzki during this colloquium.

\section{References}

Guo Y., et al., 1995, A\&A. Suppl., 112

Harmanec P., et al., 1996, A\&A, 312, 879

Hanuschik R.W., 1996, A\&A, 308, 170

Hipparcos and Tycho Catalogues, ESA SP-1200, June 1997, Vol. 12, page C38

Hoffman, J.L., et al., 1998, ApJ., 115, 1576

Labeyrie A., Proceedings of "Optical and IR Telescopes for 1990's", Vol. II, Jan. 1980, Tucson, USA, Ed. A. Hewitt, 786

Marlborough, M., 1997, A\&A, 317, L17

Mennickent R.E., Sterken C. and Vogt, 1997, A\&A, 326, 1167

Mourard, D., et al., 1989, Nature, 342, 520

Mourard, D., et al., 1994, A\&A, 283, 705

Mourard, D., et al., 1998, Proceedings of SPIE conference, Kona, Hawaii, Vol. 3350, p517

Nota, A., et al. 1995, ApJ, 448, 788

Okazaki A.T., 1997, A\&A, 318, 548

Papaloizou, J.C., et al., 1992, A\&A, 265, L45

Quirrenbach A., et al., 1997, ApJ., 479, 477

Rousselet-Perraut, et al., 1997, A\&A. Suppl., 123, 173

Smith, M., et al. ApJ, under press

SPIE conference proceedings on "Astronomical Interferometry", Kona, Hawaii, 1998, Vol. 3350

Stee, Ph., et al., 1995, A\&A, 300, 219

Stee, Ph. 1998, A\&A, 336, 980

Telting J.H., Heemsherk, et al. 1994, A\&A, 288, 558

Thom C., et al., 1986, A\&A, 165, L13

Vakili, F., et al., 1994, "Pulsation, Rotation and Mass-Loss in Early-type Stars", Proceedings of IAU Symposium 162, Eds. L. Balona et al., Kluwer Acad. Publ.

Vakili F, et al. 1997, A\&A, 323, 183

Vakili F., et al. 1998, A\&A, 335, 261

Waters, L.B.F.M., 1992, A\&A, 253, L25 